\title{
Balkanologie
}

Balkanologie Revue d'études pluridisciplinaires

Vol. IV, $n^{\circ} 2 \mid 2000$

Volume IV Numéro 2

\section{Rurbanités contemporaines en Bulgarie et en} France

\section{Krassimira Krastanova et Marine Royet}

\section{(2) OpenEdition}

\section{Journals}

Édition électronique

URL : http://journals.openedition.org/balkanologie/345

DOI : $10.4000 /$ balkanologie.345

ISSN : 1965-0582

Éditeur

Association française d'études sur les Balkans (Afebalk)

Édition imprimée

Date de publication : 1 décembre 2000

ISSN : 1279-7952

\section{Référence électronique}

Krassimira Krastanova et Marine Royet, "Rurbanités contemporaines en Bulgarie et en France », Balkanologie [En ligne], Vol. IV, nº 2 | 2000, mis en ligne le 22 avril 2008, consulté le 17 décembre 2020 URL : http://journals.openedition.org/balkanologie/345 ; DOI : https://doi.org/10.4000/balkanologie. 345

Ce document a été généré automatiquement le 17 décembre 2020.

(c) Tous droits réservés 


\title{
Rurbanités contemporaines en Bulgarie et en France
}

\author{
Krassimira Krastanova et Marine Royet
}

1 En France, est considérée comme rurale toute commune qui compte moins de cinq mille habitants agglomérés. Pour l'aménageur, l'espace rural est, au sens étymologique du terme (rus), la campagne. Il regroupe l'espace naturel - c'est-à-dire les surfaces non aménagées par l'homme - et l'espace agricole - c'est-à-dire la portion de l'espace aménagée en vue de la production agricole. On peut aussi tenter de définir le rural comme la partie de l'espace qui n'est pas urbaine.

2 Mais, de Marx à Tönnies, l'approche dichotomique ville-campagne a largement fait son temps. Elle cède la place à la thèse du continuum qui affirme que les citadins et les ruraux forment une société unique, car ils participent à une même civilisation (Mendras, 1959). Ainsi, lorsqu'on met en vis-à-vis la ville et la campagne, si l'on relève des différences d'intensité, on ne peut toutefois parler de contraste.

3 Ce point de vue théorique est largement relayé jusqu'au tout début des années 90 par nombre de spécialistes du milieu rural. Pourtant, après le courant de pensée du « toutest-urbain " des années 70, les analyses de Mendras elles-mêmes oscillent entre le continuum et la dichotomie. Il notera d'ailleurs que les transhumances urbaines, au lieu de favoriser le continuum, entraînent le renforcement du contraste entre urbains et ruraux. Ainsi, même si les sociétés rurales sont dorénavant largement ouvertes aux communications et aux influences, on se doit de chercher à savoir si le développement de ces moyens de communication modernes sont vraiment un facteur facilitant le décentrement ou la pénétration urbaine (Kayser, 1990).

À la fin du siècle dernier, le village traditionnel se caractérise par une autarcie démographique, économique et sociale; son homogénéité culturelle et une grande diversité sociale (paysans, médiateurs, ouvriers, artisans et petit personnel administratif). L'organisation sociale est fondée sur la division des fonctions par classes d'âge et de sexe. Pour compenser les baisses d'activité saisonnières, les hommes procèdent à des migrations en laissant le soin de l'exploitation agricole aux femmes et aux personnes âgées. 
5 Après la révolution industrielle, la campagne française n'est plus exclusivement et est même de moins en moins agricole et les termes «rural ", « agricole », « campagnard " ne sont plus synonymes. Henri Mendras préfère d'ailleurs parler de société paysanne, qu'il décrit comme une collectivité locale, société d'interconnaissance, dans laquelle l'individu vit la totalité de sa sociabilité, jouissant d'une certaine autonomie au sein de la société englobante, possédant une économie particulière, où les notions de salaire, de capital, de revenu, de profit n'ont pas de sens, et dont le groupe domestique est constitué sur le mode clanique.

6 En Bulgarie comme dans les autres pays balkaniques, après la libération de 1878 , le milieu rural change dans ses formes d'organisation sociale et dans son modèle économique. Les politiques balkaniques s'orientent vers la modernisation des États, en intégrant les modèles occidentaux et centre-européens. Ce fait influe sur le développement du village bien que, lors de ce processus de changement, deux modèles s'affrontent.

7 Soit les villages reprennent le modèle social hérité de la libération du xixe siècle: l'économie est organisée selon le principe de l'autarcie, le système juridique dépend du droit coutumier et les villageois s'organisent de manière à gérer et administrer de manière autonome le village.

8 Soit l'organisation villageoise intègre le nouveau modèle d'institutions politique, économique et social: l'État étend son influence sur ces milieux ruraux, occupe une position dominante et centralisatrice et modernise l'agriculture. Le nouveau modèle agricole consiste en la gestion de petites exploitations privées gardant un niveau de technique très peu développé et un bas niveau de rentabilité. Une forme d'autarcie sociale est toutefois réactivée : la sociabilité des individus s'organise autour des liens de parenté et de voisinage, les relations sont concentrées sur le territoire du village.

9 Jusqu'à la moitié du $\mathrm{xx}^{\mathrm{e}}$ siècle, se met en place, en Bulgarie «la nation des petits paysans », une nation dans laquelle l'agriculture est avant tout extensive (en 1946, $42 \%$ des agriculteurs possèdent une exploitation de 1 à 4 hectares.)

10 L'exode rural de 1965, le plus important de ce siècle en France, produit une véritable mutation de la société globale. Avec le transfert massif de population de la campagne à la ville, on passe d'une civilisation paysanne traditionnelle à une civilisation industrielle, technicienne et urbanisée. Le principal effet de cet exode sur le milieu rural est le vieillissement de la population, qui entraîne un déséquilibre de la vie sociale et économique; la disparition de la diversité sociale, puisqu'il ne reste après l'exode plus que les moyens et les gros exploitants agricoles; et la perturbation du système de pouvoir liée à la succession pendant la période d'exode de trois générations de notables (les nobles, puis les bourgeois et gros paysans, enfin les instituteurs et les médecins). Dans les années 60 , le groupe social le plus représenté en milieu rural est encore celui des paysans.

11 Pendant ce temps-là, en Bulgarie, le nouveau régime procède à des collectivisations forcées des exploitations agricoles sur tout le territoire (1947-1957). Le gouvernement communiste créé ainsi des TKZS (Trudovo Kooperativno Zemedelsko Stopantstvo: exploitations agricoles de travail coopératif). On assiste, de fait, à la disparition des petites exploitations, à la mécanisation de l'agriculture, à l'introduction de l'agronomie et de la planification. C'est à cette période que l'on observe la plus forte migration rurale, un processus qui s'amplifie après les années 60 avec l'intervention massive de 
l'État qui poursuit un objectif central dans la politique communiste : l'industrialisation du pays.

12 C'est après cette période que l'on observe, en France et en Bulgarie, l'émergence de deux phénomènes opposés liés à deux contextes politiques bien différents.

\section{Migrations urbaines et migrations rurales}

13 Ainsi, en France au début des années 70, commence un mouvement inverse de migrations urbaines. À la faveur des brassages sociaux successifs des campagnes dans ces années-là, se diffusent en milieu rural les modèles urbains.

14 L'effet le plus notable de ces migrations urbaines est certainement, et du fait des origines sociales des nouveaux arrivants, la dispersion géographique d'un capital culturel universitaire. Ceux que l'on appelle les néo-ruraux inaugurent, après la grande période "ville industrieuse contre société rurale ", une position duale : ils demeurent des urbains tout en choisissant de vivre à la campagne. Ainsi, au début des années 80 , même si la différence se fait sentir entre ruraux et urbains du point de vue des activités culturelles, les habitants des campagnes adoptent les mêmes modes de vie que ceux des villes. Les migrants urbains présents dans le paysage local depuis deux décennies ont installé leurs pratiques sociales et sont souvent acteurs du développement des territoires. On observe une homogénéisation des pratiques sur tout le territoire national, liée notamment à la présence des médias. Les espaces ruraux sont désenclavés physiquement par les voies et moyens de communication, et économiquement par les services qu'ils offrent au besoin contemporain de nature.

Alors qu'au même moment, en Bulgarie, se met en place une politique visant à organiser l'agriculture dans le cadre d'APK (Agrarno Promišlen Kompleks: complexes agro-industriels). Par les APK, l'État officialise la nationalisation des exploitations agricoles déjà imposée de manière détournée lors de l'organisation de la paysannerie en TKZS. L'agriculture est ainsi planifiée, centralisée, industrialisée. C'est une manière pour le Parti de poursuivre un autre objectif politique important : la modification de la stratification sociale sur le territoire national. Il s'agit de faire passer les individus d'un statut de petits propriétaires terriens à celui d'ouvriers agricoles saisonniers.

Ainsi, les zones de montagne rudes et enclavées se dépeuplent et la population vieillit, les migrants ne laissant derrière eux que les personnes âgées ; alors que les campagnes aux environs des villes s'étendent rapidement.

Dans ces espaces ruraux en expansion, le mode de vie des populations change. Les campagnes se modernisent (eau courante, électricité, etc.), les villages n'ont plus d'autonomie administrative, mais dépendent du pouvoir central. Pourtant, le mode d'organisation sociale reste basé sur les liens de parenté et de voisinage, sur l'endogamie territoriale et ce modèle s'étend même aux petites villes environnantes qui sont, depuis l'extension de ces espaces ruraux et la grande vague de migrations rurales, désormais en relation étroite avec le milieu rural. Les urbains des petites villes, comme les ruraux, vivent leur sociabilité sur un territoire restreint et circonscrit et ne développent pas de mobilités particulières. L'état d'esprit de ces individus proches des campagnes industrialisées peut se traduire par cette remarque d'un interlocuteur: «Pourquoi irions-nous dans le monde, alors que le monde vient à nous? ". 
18 Entre 1960 et 1990, on observe de fortes vagues de migrations rurales en grande partie provoquées par les actions incitatrices de l'État pour l'installation des ruraux aux périphéries des villes soit, en logeant gratuitement les individus près des grands complexes industriels ; soit, en leur permettant d'accéder à la propriété par petites mensualités étalées sur de très nombreuses années. Ces nouveaux urbains, en même temps qu'ils deviennent propriétaires dans un bloc (principe de l'Habitation à Loyer Modéré, les quartiers des blocs sont bâtis, dans les années 60, dans le cadre de la politique de reconstruction et de re-planification de l'espace urbain), réduisent d'autant leurs capacités de mobilité. Cet amenuisement d'une mobilité déjà restreinte par les habitudes séculaires d'accession à la propriété privée des Bulgares, ne concerne pas spécialement les nouveaux urbains, mais l'ensemble de la population, d'autant plus que le gouvernement communiste gère de manière très stricte la mobilité des individus. Ainsi, et sauf autorisation spéciale solidement argumentée demandée aux institutions compétentes, les citoyens ne peuvent en aucun cas s'installer ailleurs que sur le lieu de leur naissance ou sur celui de leur activité professionnelle.

\section{De la ruralisation des villes bulgares à l'urbanisation du territoire français}

19 Après 1990, à la restitution des terres par le gouvernement bulgare, les nouveaux urbains préfèrent continuer à vivre en ville, plutôt que reprendre leurs activités agricoles dans leurs villages d'origine. Pour autant, les liens avec la campagne sont encore actifs, car ils utilisent les terres restituées comme un complément à leur économie domestique. Ainsi, chaque fin de semaine, ils vont au village, travaillent leurs terres pour reprendre ensuite leurs vies urbaines en début de semaine.

Aujourd'hui, on voit nettement comment s'organise le rapport ville-campagne en Bulgarie. Les intrications entre les deux espaces sont nombreuses et les raisons de ces interactions sont diverses et souvent liées à la politique poursuivie pendant de nombreuses années par le gouvernement communiste et à la situation économique actuelle.

21 Avec la transition de 1989, les interactions entre la ville et la campagne se sont consolidées, d'une part, du fait de la difficulté qu'éprouvent les individus et le régime politique actuel à surmonter, malgré une politique de privatisation croissante, le centralisme d'Etat instauré sous le communisme; et, d'autre part, du fait de la crise économique qui sévit dans le pays. La campagne représente une sorte de "valeur sûre ", puisqu'elle assure les ressources alimentaires de base dans une situation d'insécurité économique totale (1997, année d'hyper-inflation à 4 chiffres). L'économie domestique est une part indispensable à la survie des individus.

Cette situation réactive les solidarités et les liens de parenté sur les lieux d'origine, qui s'étaient relâchés du fait du départ en ville des populations rurales. Dans les nouveaux quartiers, on observe des regroupements de voisinage dans les entrées et aux abords des blocs comme on peut les observer dans les villages. Les premiers habitants des blocs, nouveaux arrivants et urbains, n'avaient pas de passé commun. Au fur et à mesure ils ont mis en place des règles organisant leur vie en communauté. Aujourd'hui, les deuxième et troisième générations d'habitants ont hérités de ces règles de vie en communauté. Le plus important de leur collaboration se matérialise dans l'autogestion 
du bloc et dans l'économie domestique. Ainsi, il n'est pas rare de voir les individus préparer ensemble les conserves pour l'hiver devant l'entrée de leur bloc.

Les nouveaux arrivants sont vus par les urbains comme des individus porteurs des traditions encore actives au village. Il semblerait que les coutumes et les traditions du village aient été importées par les nouveaux arrivants dans la culture urbaine, et cela se remarque particulièrement dans les manières d'organiser les fêtes et les cérémonies et dans les modes d'accueil dans l'espace privé.

En ville, les nouveaux arrivants se sont appropriés les "espaces verts" (abords de fleuves, de lignes de chemin de fer, les espaces autour des blocs) pour cultiver les produits de grande consommation, cette appropriation a bien sûr changé notablement le paysage urbain.

Dans le même temps, le territoire français est travaillé par les circulations et les brassages sociaux et le territoire ne cesse de s'urbaniser, non pas par la croissance de ses villes, mais par le franchissement de la limite ville-campagne.

Qu'en est-il aujourd'hui de l'opposition entre la ville en mouvement et la campagne, territoire de la tradition?

On assiste à l'éclatement des limites de la ville et au passage de l'action collective à l'acte spontané, puis à l'initiative individuelle. Les contributions des néo-ruraux à une re-considération de l'espace rural sont nombreuses (visibilité sociale, mise en évidence des mutations de l'agriculture qui devient une valeur positive, revitalisation du local, nouvelle lecture spatiale allant de pair avec une plus grande mobilité); et c'est ce qui fait dire à Jean Viard que le sol de "nos vieilles terres» est partagé entre trois fonctions : la ville, la campagne, la nature (Viard, 1990).

Pourtant, parallèlement à l'effacement des différences, et du fait de la conformation des campagnes au modèle urbain, se développe l'opposition entre le local et le global, opposition que l'on voudrait dialectique. Les dynamiques de développement des territoires prennent en compte le local pour l'intégrer à la dimension globale, les projets visent à la création d'une spécificité permettant l'adaptation à la société globale.

Et cela est le résultat de deux mouvements qui successivement ont contribué à forger les représentations actuelles des Français et, de fait, les rapports qu'ils entretiennent avec ces trois espaces : la ville, la campagne, la nature.

\section{De la sacralisation de la nature aux prémices d'un mouvement écologique}

La conscience écologique émerge, en France, dans les années 60. Avec elle, l'individu prend conscience que la nature n'est pas le complément de l'homme moderne, mais qu'elle est beaucoup plus que cela. Derrière la conscience écologique, se cache le mouvement écologique, qui milite pour imposer l'idée que le maintien d'équilibres naturels et humains est indispensable à la société moderne. Depuis le début des années 90 et après bien des difficultés liées à la manière dont il s'est constitué, le mouvement écologique ne se cache plus, il fait son entrée en politique et nous voyons bien aujourd'hui, tant à propos des initiatives prises au niveau local qu'au travers des 
mesures gouvernementales, tout le poids que l'écologie peut avoir sur la conscience collective.

C'est par son filtre que l'on regarde d'un autre oeil les espaces non urbanisés au point d'en faire des réserves, des parcs ou des arrêtés de biotope. Les écologues (grands prêtres? nouveaux gourous?) tentent d'imposer ces lieux comme des espaces de protection, d'amour avec, comme point focal de religiosité, la peur de la mort... des forêts, de la couche d'ozone (Viard, 1990). C'est par son influence que l'on vient régulièrement prendre le bol d'air pur qui nous manque au coeur des villes. C'est lui qui nous pousse à redécouvrir - à réinventer - les produits locaux, les aliments cuits au feu de bois, les fibres naturelles ; à préférer le blanc cassé, les matériaux bruts et à redouter les bombes aérosols, les alertes à la pollution et la surpopulation des villes.

Et c'est bien en contribuant à l'émergence des doutes sur l'effet totalement bénéfique de l'industrialisation que le mouvement écologique a rapproché des pratiques urbaines l'offre rurale au point que, dans certains cas, elles se confondent l'une l'autre. C'est un mouvement globalisant : l'écologie concerne la ville, la campagne, le pays, l'Europe, la planète toute entière.

D'ailleurs, à l'aube de l'an 2000, un nouveau sujet d'inquiétude s'installe, il met en question l'identité nationale, ébranle l'État-nation, on l'appelle mondialisation. La mondialisation, on le constate tous les jours en prenant des nouvelles du monde, peutêtre traitée en ennemie potentielle, tant parce qu'elle met en danger la démocratie, que parce qu'elle ne tient pas compte des identités multiples. Le local devient alors une réponse aux peurs des populations de perdre à jamais leurs particularismes au profit d'une identité planétaire et la campagne est considérée comme un des derniers bastions d'identités originales. Préservée jusqu'à ces dernières décennies des influences massives, présentée bien souvent comme immobile depuis des siècles, elle semble être encore le lieu où persistent les traces des différentes étapes de l'histoire du pays; la garante des traditions françaises et l'espace d'expression de l'authenticité. L'individu va chercher sur ces territoires «naturels » ses racines, non pas les traces qu'auraient laissés d'improbables ancêtres, mais celles de l'homme premier. La "nature sauvage " est le prolongement du jardin d'Eden et chaque homme est en droit de la revendiquer comme son berceau.

En Bulgarie, l'écologie est un élément nouveau dans la vie des individus. En mars 1989, se crée le «Mouvement Écologique de Défense de Ruse », ville danubienne polluée par l'industrie chimique roumaine. Ce mouvement est créé par des intellectuels qui représentaient déjà la dissidence sous le régime communiste. En 1990, est créé le « Parti Vert » qui défend l'idée de pureté tant dans la nature que dans la vie politique. Ce parti a rallié beaucoup d'individus de la tranche d'âge des 20-40 ans. Ces deux événements représentent les prémices de l'émergence d'un mouvement écologique, et seront largement relayés par les médias et les autres organisations politiques.

À la suite de ce début politique, des O.N.G. dont l'objectif est la défense de l'environnement et dont la mission est de faire des propositions dans ce domaine, sont créées dans les grandes villes. Leurs actions sont souvent très concrètes, puisqu'elles réunissent les individus autour de projets de nettoyage des abords des rivières ou des montagnes. L'idée principale qu'elles véhiculent est qu'en sauvegardant la nature, on sauvegarde la planète entière. C'est ainsi que le projet de construction d'une nouvelle centrale nucléaire sur le Danube sera porté et discuté dans l'espace public en 1992 et 
que les associations de sauvegarde de l'environnement joueront un grand rôle dans l'abandon du projet.

C'est le premier pas dans la prise de conscience des individus de l'importance de sauvegarder la planète pour les générations futures.

\section{D'espaces protégés en espaces protégés}

Avant 1989, existaient déjà quelques réserves naturelles dont l'objectif était de préserver la nature et de sauvegarder les espèces animales en voie de disparition. Ces réserves n'avaient toutefois pas entraîné l'édition de lois particulières ni l'élaboration de cahiers des charges permettant d'appliquer des règles données sur des territoires définis. Les lieux à protéger étaient simplement décrétés tels par le gouvernement bulgare.

Depuis quelques années, le Ministère de l'Environnement a édicté des lois pour les Parcs nationaux, les jardins municipaux et les réserves appelant une protection. Ces zones sont désormais sous le contrôle de l'État.

Il est intéressant de noter que toutes les lois publiées par le Ministère de l'Environnement, ainsi que les zones de protection répertoriées n'ont pas donné lieu à publicité, l'information n'a pas été soutenue par les médias, ainsi, seules les personnes bien informées connaissent ces dispositions prises il y a quelques années par l'actuel gouvernement. Pour la grande majorité des Bulgares, ces dispositions en faveur de zones protégées restent très éloignées de leur quotidien voire ignorées.

La conscience écologique ne s'est pas répandue par le biais du mouvement du même nom ou des initiatives gouvernementales, en revanche cette prise de conscience se fait, dans une certaine mesure et pour les zones concernées, par l'intermédiaire du tourisme. Les individus, particulièrement ceux qui vivent dans les régions touristiques, commencent à considérer que la valeur ajoutée du pays viendra de l'entretien de l'environnement.

41 En France, c'est bien le nouveau rôle social que joue la campagne face à la déréliction du monde, les tendances à la sacralisation de l'espace Nature et cette nouvelle forme "d'urbanisation généralisée » qui font dire à J. Viard que nous vivons une époque de bouleversement des anciens rapports ville-campagne par l'émergence de ce nouvel espace protégé de l'homme par l'homme, l'espace Nature, qu'il nomme le tiers espace résultat ou contribution à la naissance d'une nouvelle société occupant par sa grande mobilité ces trois espaces à la fois (Viard, 1990). Une société des trois pôles que l'on ne peut qualifier seulement de rurale ou d'urbaine.

Ce tiers espace est souvent matérialisé, en France, par les Parcs naturels régionaux, espaces fréquentés par tous : lieu de résidence pour certains et terrain de jeux pour d'autres. Conçus pour répondre à la demande sociale de Nature, ils se présentent souvent comme de poumons verts, en périphérie des villes. Les Parcs naturels régionaux français présentés comme des territoires de développement local, comme des lieux de sauvegarde de la nature, de préservation et d'animation du patrimoine culturel, sont surtout gérés de manière à attirer le plus possible les flux des populations environnantes, voire au-delà, par l'offre de services touristiques. Par le fait de ce double jeu, il leur est difficile de trouver une légitimité. Que sont-ils en fait? Des 
appareils de protection de la nature et d'aide au développement des sociétés résidentes? Des zones de concentration touristique?

Ils semblent être, en tous cas, le territoire le plus adapté aux modes de vie et aux représentations des individus qui forgent leur identité dans la polyspatialité. Par un mouvement de fragmentation du monde, par lequel chaque espace devient monofonctionnel, l'individu redéfinit des règles de partage spatial pour se construire un espace qui lui est propre, à partir duquel il construit une identité originale qui n'est plus en correspondance avec un territoire physique que l'on peut circonscrire géographiquement. Pour l'existence de ces individus, tous les lieux sont nécessaires : la ville parce qu'elle représente le pôle des activités culturelles et professionnelles, la campagne parce qu'elle est le lieu du ressourcement garantissant une certaine qualité de vie propice à la vie de famille et la nature parce qu'elle devient le lieu de l'appartenance originelle. Et bien sûr, aucun des trois n'est suffisant.

Ces nouvelles manières d'habiter le monde qui remettent en question le mode classique d'appréhension de l'espace, sont rendues possibles avant tout par la diffusion des effets de la modernisation et par l'enrichissement de l'ensemble de la société.

Depuis les années 50, en Bulgarie comme en France, la dichotomie ville / campagne est largement remise en question par les mouvements successifs de populations. Pourtant, aujourd'hui, nous ne pouvons que constater que les schémas d'analyse opérants sur le territoire français ne le sont pas totalement en ce qui concerne la Bulgarie. Nous devons chercher les raisons des effets inverses des mouvements de populations ayant eu lieu dans les mêmes périodes de ce siècle dans les situations politiques, idéologiques et économiques bien différentes des deux pays.

Ainsi, en France, alors que la société moderne et industrielle est remise en cause au début des années 70 , on observe des migrations urbaines qui ont dans un premier temps, comme conséquence, l'urbanisation des milieux ruraux, puis, dans un second temps, la sacralisation de l'espace-Nature.

Alors que la Bulgarie vit, à la même époque, une industrialisation massive du pays qui entraîne des migrations rurales et la conséquence inverse de ruralisation des villes qui aujourd'hui, amène à l'émergence timide d'un nouveau rapport à la nature. Dans ce contexte historique, politique et économique, la campagne est avant tout considérée de nos jours comme un "garde-manger " et un modèle en matière de sociabilité avant d'être intégrée dans les représentations des individus comme un espace à protéger et à réinventer.

\section{INDEX}

Index géographique : Bulgarie, France 